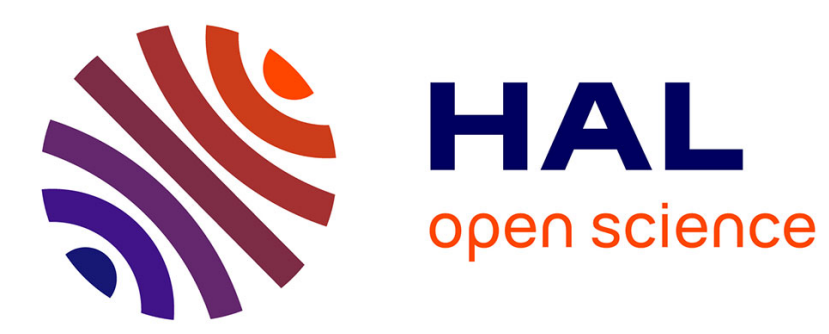

\title{
On Stability of Measure Driven Differential Equations
}

\author{
Aneel Tanwani, Bernard Brogliato, Christophe Prieur
}

\section{To cite this version:}

Aneel Tanwani, Bernard Brogliato, Christophe Prieur. On Stability of Measure Driven Differential Equations. NOLCOS 2013 - 9th IFAC Symposium on Nonlinear Control Systems, Sep 2013, Toulouse, France. pp.241-246, 10.3182/20130904-3-FR-2041.00135 . hal-00926112

\section{HAL Id: hal-00926112 \\ https://hal.science/hal-00926112}

Submitted on 9 Jan 2014

HAL is a multi-disciplinary open access archive for the deposit and dissemination of scientific research documents, whether they are published or not. The documents may come from teaching and research institutions in France or abroad, or from public or private research centers.
L'archive ouverte pluridisciplinaire HAL, est destinée au dépôt et à la diffusion de documents scientifiques de niveau recherche, publiés ou non, émanant des établissements d'enseignement et de recherche français ou étrangers, des laboratoires publics ou privés. 


\title{
On Stability of Measure Driven Differential Equations
}

\author{
Aneel Tanwani* Bernard Brogliato* Christophe Prieur ** \\ * Team BipOp, INRIA Rhône-Alpes, 655 Avenue de l'Europe, \\ 38334 Montbonnot Cedex, France. \\ Email: $\{$ aneel.tanwani, bernard.brogliato\}@inria.fr \\ ** Gipsa-lab, Department of Automatic Control, 11 Rue des \\ Mathématiques, BP 46, 38402 Saint Martin d'Hères, France. \\ Email: christophe.prieur@gipsa-lab.fr
}

\begin{abstract}
We consider the problem of stability in a class of differential equations which are driven by a differential measure associated with the inputs of locally bounded variation. After discussing some existing notions of solution for such systems, we derive conditions on the system's vector fields for asymptotic stability under a specific class of inputs. These conditions present a trade-off between the Lebesgue-integrable and the measure-driven components of the system. In case the system is not asymptotically stable, we derive weaker conditions such that the norm of the resulting trajectory is bounded by some function of the total variation of the input, which generalizes the notion of integral input-to-state stability in measure-driven systems.
\end{abstract}

\section{INTRODUCTION}

In the theory of dynamical systems, a large class of nonlinear systems are modeled by the following ordinary differential equation:

$$
\frac{d x}{d t}(t)=f(x(t))+g(x(t)) u(t)
$$

where $f, g: \mathbb{R}^{n} \rightarrow \mathbb{R}^{n}$ are the vector fields and $u$ : $\left[t_{0}, \infty\right) \rightarrow \mathbb{R}$ is the scalar input. If $f(\cdot)$ and $g(\cdot)$ are locally Lipschitz then there exists an interval over which system (1) admits a unique absolutely continuous solution $x(\cdot)$ for every Lebesgue-measurable function $u(\cdot)$, where the resulting solution satisfies (1) almost everywhere (with respect to the Lebesgue measure) on the interval where the solution exists. Under further regularity assumptions, say $f(\cdot), g(\cdot)$ satisfy a linear growth condition, the solution is defined for all times, in which case we say that $f(\cdot), g(\cdot)$ are forward complete.

In this paper, we consider measure differential equations (MDEs) modeled as:

$$
d x=f(x) d t+g(x) d u .
$$

Just like (1) is driven by an input $u(\cdot)$, the dynamics of system (2) can be seen as driven by the "derivative" of $u(\cdot)$. However, to achieve generality, we assume that $u(\cdot)$ is a right-continuous function with locally bounded variation (locally $R C B V$ ), so its generalized derivative, or the so-called differential measure, is represented by $d u$. If $u(\cdot)$ is absolutely continuous, then the derivative of $u(\cdot)$ is a Lebesgue-measurable function. In that case, we take $d u=\dot{u} d t$, where $\dot{u}$ represents the classical derivative, and system (2) is equivalent to (1) with $u(\cdot)$ replaced by $\dot{u}(\cdot)$.

The framework of system (2) can be used to model the dynamics of a rich class of state trajectories. If $u(\cdot)$ is considered to be a piecewise constant function, then $d u$ is a sum of Dirac impulses, in which case (2) models discontinuities in the state trajectory. Another advantage of considering MDEs is that we can possibly model Zeno trajectories because $u(\cdot)$ as a function of bounded variation may exhibit infinitely many discontinuities in a finite interval. This also makes the simulation of Zeno trajectories possible as one no longer uses the classical eventbased schemes to model discontinuities but rather a timestepping algorithm is employed as demonstrated in Acary et al. [2008]. Other than the discontinuities, singularly continuous inputs (such as a random process with continuous sample path) may also be considered. Certain applications of such mathematical models for modelling of mechanical systems are discussed in Brogliato [1999].

The solution theory for such systems could be traced back to the work of Kurzweil [1958], where he approximated the integral version of (2) with a sequence of integrals associated with ordinary differential equations to develop existence conditions for a generalized solution. In the context of optimal control, measure differential equations were studied by Schmaedeke [1965], where he also generalized Caratheodory's concept of solution for systems of form (2) with $g(\cdot)$ being time-dependent only. Sussmann [1978] studied such differential equations to develop solution concept for a class of stochastic differential equations using the theory of ordinary differential equations, but only considered singularly continuous inputs. Based on his work, Bressan [1987] and later Bressan Jr. and Rampazzo [1991] generalized the idea to consider discontinuous inputs, and in general, the functions of bounded variation.

From stability point of view, the work of Code and Silva [2010] talks about stabilization in terms of existence of an input function that makes some Lyapunov function decrease along the trajectories of the system (2).

In our work, we will adopt the solution framework proposed by Sussmann [1978] and later developed by Bressan Jr. and Rampazzo [1991]. A brief account of this solution methodology is collected in Section 3 which we 
would also use in deriving conditions for stability of the system. Our approach towards the problem of stability is different from any of the work mentioned before. We study two different notions of stability for system (2) and provide related sufficient conditions. The first one relates to the convergence of the state trajectories towards the equilibrium. Since the stability would in general be affected by the inputs, in the proposed sufficient condition, we specify a class of inputs for which the system admits asymptotically convergent behavior.

The other notion of stability considered in this paper relates to input-to-state stability (ISS) pioneered by Sontag [1989]. The basic idea is to study weather the norm of the state trajectories is bounded by some increasing function of the norm of the input. In this regard, for system (2), we compare the supremum norm of the state trajectories with the total variation of the input. Since the total variation of a function equals the integral of the absolute value of the derivative of the function (when the function is differentiable), the considered problem is a generalization of the integral variant of ISS studied in Sontag [1998].

\section{PRELIMINARIES}

We recall some preliminary results related to functions of bounded variation which will be used later on. For a function $f:[s, t] \rightarrow \mathbb{R}^{n}$, the total variation is a non-decreasing function and is defined for $\tau \in[s, t]$ as $\operatorname{var}_{f}(\tau)=\sup \sum_{i=1}^{k}\left|f\left(t_{i}\right)-f\left(t_{i-1}\right)\right|$, where the supremum is taken over all integers $k$, and all possible choices of the sequence $\left\{t_{i}\right\}$ such that $s=t_{0}<t_{1}<\cdots<t_{k}=\tau$. The function $f(\cdot)$ is said to be of bounded variation $(B V)$ on $[a, b]$ if $\operatorname{var}_{f}(b)<\infty$. If it is right continuous with bounded variation we denote it as $R C B V$. It is locally $R C B V$ if this holds for any compact interval $[a, b] \subset \mathbb{R}$. If $f(\cdot)$ is $B V$ on $[a, b]$ then it has at most a countable number of jump discontinuities. Moreover, it has right and left limits everywhere. The right and left limits of a function at $t$ are denoted as $f\left(t^{+}\right):=\lim _{s \searrow t} f(s)$ and $f\left(t^{-}\right):=\lim _{s \nearrow t} f(s)$, respectively, and are well defined for $B V$ functions. In this notation, right continuity of $f(\cdot)$ in $t$, means that $f\left(t^{+}\right)=f(t)$.

For an interval $I \subseteq \mathbb{R}$, we denote by $\mathcal{L}^{1}\left(I, \mathbb{R}^{n} ; \nu\right)$ and $\mathcal{L}_{\text {loc }}^{1}\left(I, \mathbb{R}^{n} ; \nu\right)$ the space of integrable and locally integrable functions, respectively, from $I$ to $\mathbb{R}^{n}$ with respect to the measure $\nu$. If the measure is not specified then the integration is with respect to the Lebesgue measure. Similarly, $\mathcal{C}^{1}\left(\mathbb{R}, \mathbb{R}^{n}\right)$ denotes the set of continuously differentiable functions from $\mathbb{R}$ to $\mathbb{R}^{n}$.

For a non-decreasing, right-continuous function $u(\cdot)$ of bounded variation, we can associate a Lebesgue-Stieltjes measure $\mu=d u$ such that

$$
\mu((s, t])=u(t)-u(s) .
$$

It is well-known that every $R C B V$ function could be written as a difference of two right-continuous non-decreasing functions, that is, there exist some functions $u^{+}$and $u^{-}$ (both non-decreasing) such that $u=u^{+}-u^{-}$. We can then associate the Lebesgue-Stieltjes measure with each of these functions and denote them by $\mu^{+}$and $\mu^{-}$, respectively. Thus, if $\mu$ denotes the Lebesgue-Stieltjes measure associated with an $R C B V$ function $u(\cdot)$, then $\mu=\mu^{+}-\mu^{-}$.
The total variation of this measure is denoted by $|\mu|$, and is equal to $\mu^{+}+\mu^{-}$. We will use the notation $|\mu|_{\left[t_{0}, t\right]}$ to denote the total variation of the measure $\mu$ over the interval $\left[t_{0}, t\right]$ and it is seen that $|\mu|_{\left[t_{0}, t\right]}=\operatorname{var}_{u}(t)$.

The distributional derivative $d u$ (also called the differential measure) introduced in (2) could be seen as a generalized notion of derivative and satisfies the following relation:

$$
\mu((s, t])=u(t)-u(s)=\int_{(s, t]} d u .
$$

One may verify that, for absolutely continuous functions, if we let $d u=\dot{u} d t$ with $\dot{u}(\cdot)$ a Lebesgue-measurable function denoting the classical derivative of $u(\cdot)$, then the above relation is obtained simply from the fundamental theorem of calculus. It is noted that the $R C B V$ function $u(\cdot)$ is decreasing (or, non-decreasing) over an interval if $d u<0$ (resp., $d u \leq 0$ ).

We also recall that the function $u(\cdot)$, being locally $R C B V$, has countably many discontinuities over the interval $\left[t_{0}, \infty\right)$, and we let $\mathcal{I}$ denote a countable set that indexes the discontinuities of $u(\cdot)$. The measure $\mu$ associated with $u(\cdot)$ admits the following decomposition: $\mu=\mu_{\mathrm{ac}}+$ $\mu_{\text {sing }}+\mu_{d}$, where $\mu_{\text {ac }}$ is absolutely continuous with respect to Lebesgue measure, $\mu_{\text {sing }}$ is associated with a singular function which is continuous everywhere and differentiable almost everywhere with zero derivative (e.g., the Cantor function). We use the notation $\mu_{c}:=\mu_{\mathrm{ac}}+\mu_{\text {sing }}$ to denote the continuous part of $\mu$. The discontinuous part is given by $\mu_{d}:=\sum_{i \in \mathcal{I}} \mu\left(\left\{t_{i}\right\}\right)$, where $\mu\left(\left\{t_{i}\right\}\right)$ denotes the jump discontinuity at time $t_{i}$. To clarify some notation later and better understand the definition of the measure $\mu$, note that if $u(\cdot)$ is $R C B V$ on an interval $[s, t]$ with a single discontinuity at $t_{i}, s<t_{i}<t$, then $\mu_{d}([s, t])=\mu\left(\left\{t_{i}\right\}\right)=$ $u\left(t_{i}^{+}\right)-u\left(t_{i}^{-}\right)$, and $\mu_{c}([s, t])=\mu([s, t])-\mu_{d}([s, t])$.

\section{SOLUTION CONCEPTS}

As stated in the introduction, the solution of system (2) is well-defined for an absolutely continuous input $u(\cdot)$. However, in this paper, it is assumed that the function $u(\cdot)$ is only locally $R C B V$ in which case the term $d u$ denotes the differential measure associated with $u(\cdot)$. When $g(\cdot)$ is assumed to be a continuous function of time only, and not of state, there is no ambiguity in the interpretation of the solution. In that case, Carathédory's notion of solution for ordinary differential equation has been extended to arrive at similar conditions for existence and uniqueness of solution to system (2) in the class of locally $R C B V$ functions, see Schmaedeke [1965]. However, with the vector field $g(\cdot)$ considered as a function of the state variable, the notion of solution is not so straight forward. The following example illustrates some degree of difficulty in obtaining the solution to MDE (2).

Example 1. Consider system (2) with $f(x)=0, g(x)=$ $x$, and $u(t)=c_{1} H(t)$, where $c_{1}>0$, and $H(t)=$ $\left\{\begin{array}{ll}0, & t_{0}<t<t_{1} \\ 1, & t_{1} \leq t<\infty\end{array}\right.$. This gives $d u=c_{1} \delta_{t_{1}}$, where $\delta_{t_{1}}$ denotes the Dirac impulse at time $t_{1}$. It is clear that $x(t)=$ $x\left(t_{0}\right)$, for $t_{0} \leq t<t_{1}$. For $t \geq t_{1}$, if we pick $x(t)=$ $\left(1+c_{1}\right) x\left(t_{0}\right)$ then the equation $\int_{\left\{t_{1}\right\}} d x=x\left(t_{1}^{-}\right) \int_{\left\{t_{1}\right\}} d u$ holds; however, the solution $x(t)=x\left(t_{0}\right) /\left(1-c_{1}\right)$ satisfies 
$\int_{\left\{t_{1}\right\}} d x=x\left(t_{1}^{+}\right) \int_{\left\{t_{1}\right\}} d u$. Another solution is obtained by approximating $u(\cdot)$ with a sequence $\left\{u_{k}\right\}_{k=1}^{\infty}$ of continuously differentiable functions. Then for each element of the sequence, the resulting solution is obtained by solving $\frac{\dot{x}_{k}}{x}=\dot{u}_{k}(t)$, which leads to $x_{k}(t)=x\left(t_{0}\right) e^{u_{k}(t)}$. One then takes $x(\cdot)$ to be the limit of the sequence $\left\{x_{k}\right\}_{k=1}^{\infty}$ and let $x(t)=x\left(t_{0}\right) e^{u(t)}$ to be the solution. In terms of the original system description in differential form, this last solution satisfies $\int_{\left\{t_{1}\right\}} d x=\tilde{x} \int_{\left\{t_{1}\right\}} d u$, for some $\tilde{x} \in\left[x\left(t_{1}^{-}\right), x\left(t_{1}^{+}\right)\right]$.

It is seen that the first two notions of solutions considered in the example are not continuous with respect to the inputs. In general, such solutions won't be unique either. However, the last solution obtained by approximating $u(\cdot)$ with continuously differentiable functions has some nice properties and we will develop results on stability with respect to such solutions. Formally, the solution to system (2) is defined as follows:

Definition 1. For a given locally $R C B V$ input $u:\left[t_{0}, \infty\right) \rightarrow$ $\mathbb{R}$, a locally $R C B V$ function $x(u, \cdot):\left[t_{0}, \infty\right) \rightarrow \mathbb{R}^{n}$ is called a solution of (2) if there exists a sequence of controls $u_{k} \in \mathcal{C}^{1}\left(\left[t_{0}, \infty\right), \mathbb{R}\right)$ such that $u_{k}\left(t_{0}\right)=u\left(t_{0}\right), u_{k} \rightarrow u$ in $\mathcal{L}^{1}$-norm on every compact interval $I \subset\left[t_{0}, \infty\right)$ and the corresponding trajectories $x\left(u_{k}, \cdot\right) \rightarrow x(u, \cdot)$ in $\mathcal{L}^{1}$-norm on $I$.

In order to prove the existence of solution in the sense of Definition 1, we recall the work of Sussmann [1978] and Bressan Jr. and Rampazzo [1991]. Towards that end, let $\Phi_{g}\left(s ; x_{0}\right)$ denote the Carathéodory solution of the ordinary differential equation $\dot{x}=g(x)$ at time $s$ with initial condition $x_{0}$, i.e., $\Phi_{g}\left(0 ; x_{0}\right)=x_{0}$. It is seen that $\Phi_{g}^{-1}\left(s ; x_{0}\right)=\Phi_{g}\left(-s ; x_{0}\right)$. Now, consider the state transformation $\varphi: \mathbb{R}^{n+1} \rightarrow \mathbb{R}^{n+1}$ defined as:

$$
\bar{y}:=\left(y, y_{u}\right):=\varphi(x, u):=\left(\Phi_{g}(-u ; x), u\right) .
$$

The system dynamics in these coordinates are then given by:

$$
\begin{aligned}
\dot{y} & =F\left(y, y_{u}\right), & y\left(t_{0}\right) & =\Phi_{g}\left(-u\left(t_{0}\right) ; x\left(t_{0}\right)\right), \\
d y_{u} & =d u, & y_{u}\left(t_{0}\right) & =u\left(t_{0}\right) .
\end{aligned}
$$

where $F\left(y, y_{u}\right)=\left.\frac{\partial \Phi_{g}(-u, x)}{\partial x}\right|_{(x, u)=\left(\Phi_{g}\left(y_{u} ; y\right), y_{u}\right)} \cdot f\left(\Phi_{g}\left(y_{u} ; y\right)\right)$. In (4), it is important to note that there is no discontinuous function multiplying the measure $d u$, which makes it easier to study the solution of this transformed system.

To formally prove the existence of solution to system (2) in the sense of Definition 1, a sequence of solutions $\left\{\bar{y}_{k}\right\}$ is first obtained from continuously differentiable functions $u_{k}$. The limit of the sequence $\left\{\bar{y}_{k}\right\}$, let's say $\bar{y}$, is then mapped under the map $\varphi^{-1}$ to obtain the solution $x(\cdot)$. We thus have the following result:

Proposition 2. Assume that the equation (4a) has a solution on every compact interval $\left[t_{0}, T\right]$, then

$$
x(t)=\Phi_{g}(u(t) ; y(t)), \quad \forall t \in\left[t_{0}, T\right]
$$

is the solution of (2) in the sense of Definition 1.

It is important to note that the forward completeness of the vector fields $f(\cdot)$ and $g(\cdot)$ is not sufficient to guarantee that the solution of $(4 \mathrm{a})$ is forward complete. While working with continuous inputs, a counterexample has been given in [Sussmann, 1978, Section 8]. In the same paper in Theorem 2, he presented sufficient conditions (which roughly state that $f(\cdot)$ satisfies a linear growth condition and $g(\cdot) \in \mathcal{C}^{1}\left(\mathbb{R}^{n}, \mathbb{R}^{n}\right)$ with $\frac{\partial g}{\partial x}$ uniformly bounded) that guarantee the existence and uniqueness of the solutions to (4) with $u(\cdot)$ being continuous. Later, it was shown in [Bressan and Rampazzo, 1993, Theorem 2.1] that these conditions are sufficient for existence of global (in time) solutions of (4a). Without imposing such conditions on system vector fields, we work under the following assumption in the remainder of the paper so that the solution of system (2) is considered according to Proposition 2.

Assumption 1. There exists a unique solution to the ordinary differential equation (4a) on every compact interval for each $R C B V$ function $y_{u}(\cdot)$.

An interesting outcome of the coordinate transformation introduced in (3) is that the solution $x(\cdot)$ at the discontinuities is characterized as follows:

Proposition 3. At the atoms $\left\{t_{i}\right\}$ of $\mu$, we have

$$
x\left(t_{i}^{+}\right)=\Phi_{g}\left(u\left(t_{i}^{+}\right)-u\left(t_{i}^{-}\right) ; x\left(t_{i}^{-}\right)\right) .
$$

To see this, note that from Proposition 2 we have, $x\left(t_{i}^{+}\right)=\Phi_{g}\left(u\left(t_{i}^{+}\right) ; y\left(t_{i}^{+}\right)\right)$, where $y\left(t_{i}^{+}\right)=y\left(t_{i}^{-}\right)=$ $\Phi_{g}\left(-u\left(t_{i}^{-}\right) ; x\left(t_{i}^{-}\right)\right)$. Since $\Phi_{g}$ satisfies the group action properties, we get

$\Phi_{g}\left(u\left(t_{i}^{+}\right) ; \Phi_{g}\left(-u\left(t_{i}^{-}\right) ; x\left(t_{i}^{-}\right)\right)\right)=\Phi_{g}\left(u\left(t_{i}^{+}\right)-u\left(t_{i}^{-}\right) ; x\left(t_{i}^{-}\right)\right)$.

Revisiting Example 1: To get a good understanding of the foregoing discussion, let us apply the coordinate transformation in (3) to the system considered in Example 1. In that case, $\Phi_{g}(-u ; x)=x e^{-u}$, so that

$$
\bar{y}=\varphi(x, u)=\left(x e^{-u}, u\right) .
$$

Since, $f(x) \equiv 0$, we simply get

$$
\dot{\bar{y}}=\left[\begin{array}{cc}
e^{-u} & -x e^{-u} \\
0 & 1
\end{array}\right]\left(\begin{array}{l}
x \\
1
\end{array}\right) d u=\left(\begin{array}{l}
0 \\
1
\end{array}\right) d u \text {. }
$$

Thus, $y(t) \equiv y\left(t_{0}\right)=x\left(t_{0}\right) e^{-u\left(t_{0}\right)}$ remains constant, whereas $y_{u}(t)=u(t)$. Applying the inverse transformation, we get $x(t)=e^{u(t)-u\left(t_{0}\right)} x\left(t_{0}\right)$, which coincides with our earlier observation.

\section{STABILITY RESULTS}

We now study the stability properties of system (2) with respect to the origin. It is assumed that $f(0)=0$, so that the origin is indeed an equilibrium point of the unforced system. Two variants of the stability notions will be considered. The first one relates to the attractivity of the state trajectories towards the origin for a specific class of inputs and is studied in Section 4.1. Then, in Section 4.2, we derive weaker conditions to establish the boundedness of the state trajectories in terms of the total variation of the input.

Before stating the main result, we recall some standard definitions: A function $\alpha:[0, \infty) \rightarrow[0, \infty)$ belongs to class $\mathcal{K}$ if it is continuous, strictly increasing, and $\alpha(0)=0$. If, in addition, $\alpha$ is unbounded then $\alpha$ belongs to class $\mathcal{K}_{\infty}$. A function $\beta:[0, \infty) \times[0, \infty) \rightarrow[0, \infty)$ belongs to class $\mathcal{K} \mathcal{L}$ if $\beta(\cdot, t) \in \mathcal{K}$ for each $t \geq 0, \beta(r, \cdot)$ is strictly decreasing for each $r \geq 0$ and $\beta(r, t) \rightarrow 0$ as $t \rightarrow \infty$. 


\subsection{Asymptotic Stability}

We call the system (2) uniformly asymptotically stable over a set of inputs $\mathcal{U}$ if there exists a class $\mathcal{K} \mathcal{L}$ function $\beta$ such that every state trajectory $x(\cdot)$ resulting from the input $u \in \mathcal{U}$ as a solution of (2) satisfies $|x(t)| \leq \beta\left(\left|x\left(t_{0}\right)\right|, t-t_{0}\right)$. Theorem 4. Assume that there exists a continuously differentiable function $V: \mathbb{R}^{n} \rightarrow \mathbb{R}_{+}$, some class $\mathcal{K}_{\infty}$ functions $\alpha_{1}, \alpha_{2}$, and some constants $a, \underline{b}, \bar{b} \in \mathbb{R}$ such that the following holds for each $x \in \mathbb{R}^{n}$ :

$$
\begin{aligned}
& \alpha_{1}(|x|) \leq V(x) \leq \alpha_{2}(|x|), \\
& \langle\nabla V(x), f(x)\rangle \leq a V(x), \\
& \underline{b} V(x) \leq\langle\nabla V(x), g(x)\rangle \leq \bar{b} V(x) .
\end{aligned}
$$

Let $\mathcal{U}_{c, \theta}$ denote the class of inputs satisfying:

$$
a\left(t-t_{0}\right)+\bar{b} \mu^{+}\left(\left[t_{0}, t\right]\right)-\underline{b} \mu^{-}\left(\left[t_{0}, t\right]\right) \leq c-\theta\left(t-t_{0}\right)
$$

for some $c>0$ and $\theta \in \mathcal{K}_{\infty}$. Then, system (2) is uniformly asymptotically stable over $\mathcal{U}_{c, \theta}$.

Proof. Consider the Lyapunov function $V(\cdot)$ satisfying the hypothesis in the statement of the theorem. Since $V(\cdot)$ is continuously differentiable and the solution $x(\cdot)$ is locally $R C B V$, the composition function $V(x(t))$ is also locally $R C B V$ [Moreau and Valadier, 1987, Theorem 3]. Over an interval $\left(t_{i}, t_{i+1}\right)$ between the two discontinuities of the input $u(\cdot)$, the state trajectory $x(\cdot)$ is continuous and the differential measure of $V(\cdot)$ is computed by

$$
d V=\langle\nabla V(x), f(x)\rangle d t+\langle\nabla V(x), g(x)\rangle d u
$$

Writing $u=u^{+}-u^{-}$and using (5b), (5c), we get

$$
d V \leq a V d t+\bar{b} V d u^{+}-\underline{b} V d u^{-} .
$$

For $t \in\left(t_{i}, t_{i+1}\right)$, let $m(t):=a\left(t-t_{i}\right)+\bar{b} \mu^{+}\left(\left(t_{i}, t\right)\right)-$ $\underline{b} \mu^{-}\left(\left(t_{i}, t\right)\right)$, and

$$
W(t):=e^{-m(t)} V(t) .
$$

The use of product rule and chain rule for differential of bounded variation functions (recalled from [Monteiro Marques, 1993, Page 8] and [Moreau and Valadier, 1987, Theorem 3]) and inequality (7) yield:

$$
\begin{aligned}
d W & =V(t) e^{-m(t)}\left(-a d t-\bar{b} d u^{+}+\underline{b} d u^{-}\right)+e^{-m(t)} d V \\
& \leq 0 .
\end{aligned}
$$

Thus, $W(\cdot)$ is non-increasing on the interval $\left(t_{i}, t_{i+1}\right)$ for each $i \in \mathcal{I}$, and since $W(t)$ is right-continuous, $W(t) \leq$ $W\left(t_{i}\right)$, which implies that

$$
V(t) \leq e^{m(t)} V\left(t_{i}\right) .
$$

If $\left\{t_{i}\right\}$ is an atom of the measure $\mu$, then from Proposition $3, x\left(t_{i}^{+}\right)$is obtained by the solution of the differential equation $\dot{z}(s)=g(z(s))$ at time $s=\mu\left(\left\{t_{i}\right\}\right)=\mu^{+}\left(\left\{t_{i}\right\}\right)-$ $\mu^{-}\left(\left\{t_{i}^{-}\right\}\right)$with the initial condition $z(0)=x\left(t_{i}^{-}\right)$. Equivalently, one can solve $\dot{z}(s)=g(z(s))$ for $s \in\left[0, \mu^{+}\left(\left\{t_{i}\right\}\right)\right]$ and $\dot{z}(s)=-g(z(s))$ over the interval $\left[\mu^{+}\left(\left\{t_{i}\right\}\right), \mu^{+}\left(\left\{t_{i}\right\}\right)+\right.$ $\left.\mu^{-}\left(\left\{t_{i}\right\}\right)\right]$ with $z(0)=x\left(t_{i}^{-}\right)$. Let $v(s)=V(z(s))$, then for each $s \in\left[0, \mu^{+}\left(\left\{t_{i}\right\}\right)\right], \dot{v}(s)=\langle\nabla V(z), g(z)\rangle \leq \bar{b} v(s)$, and the comparison lemma [Khalil, 2002, Lemma 3.4] yields

$$
v\left(\mu^{+}\left(\left\{t_{i}\right\}\right)\right) \leq e^{\bar{b} \mu^{+}\left(\left\{t_{i}\right\}\right)} v(0) .
$$

For the interval $\left[\mu^{+}\left(\left\{t_{i}\right\}\right), \mu^{+}\left(\left\{t_{i}\right\}\right)+\mu^{-}\left(\left\{t_{i}\right\}\right)\right]$, we have $\dot{v}(s)=\langle\nabla V(z),-g(z)\rangle \leq-\underline{b} v(s)$ which, using comparison lemma, leads to

$$
\begin{aligned}
v\left(\mu^{+}\left(\left\{t_{i}\right\}\right)+\mu^{-}\left(\left\{t_{i}\right\}\right)\right) & \leq e^{-\underline{b} \mu^{-}\left(\left\{t_{i}\right\}\right)} v\left(\mu^{+}\left(\left\{t_{i}\right\}\right)\right) \\
& \leq e^{\bar{b} \mu^{+}\left(\left\{t_{i}\right\}\right)-\underline{b} \mu^{-}\left(\left\{t_{i}\right\}\right)} v(0) .
\end{aligned}
$$

Substituting the value of $v$ and using $x\left(t_{i}^{+}\right)=z\left(\mu^{+}\left(\left\{t_{i}\right\}\right)+\right.$ $\left.\mu^{-}\left(\left\{t_{i}\right\}\right)\right)$, we get

$$
V\left(x\left(t_{i}^{+}\right)\right) \leq e^{\bar{b} \mu^{+}\left(\left\{t_{i}\right\}\right)-\underline{b} \mu^{-}\left(\left\{t_{i}\right\}\right)} V\left(x\left(t_{i}^{-}\right)\right) .
$$

Combining (8) with (9) and using the countable additivity of the measure $\mu(\cdot)$, we get:

$$
\begin{aligned}
V(t) & \leq e^{a\left(t-t_{0}\right)+\bar{b} \mu^{+}\left(\left[t_{0}, t\right]\right)-\underline{b} \mu^{-}\left(\left[t_{0}, t\right]\right)} V\left(t_{0}\right) \\
& \leq e^{c-\theta\left(t-t_{0}\right)} V\left(t_{0}\right)
\end{aligned}
$$

where we used (6) to obtain the last inequality. Using (5a) and letting $\beta(r, t):=\alpha_{1}^{-1}\left(e^{c} \alpha_{2}(r)\right) e^{-\theta(t)}$, we obtain

$$
|x(t)| \leq \beta\left(\left|x\left(t_{0}\right)\right|, t-t_{0}\right),
$$

which proves the desired result since $\beta \in \mathcal{K} \mathcal{L}$.

Remark 5. For impulsive systems, the result of Theorem 4 could be seen as a generalization of [Hespanha et al., 2008, Theorem 1] where the authors give conditions on frequency of impulsive times depending on margin of stability/instability in continuous and discrete dynamics.

We now address a special case of Theorem 4 for a specific class of inputs. Let $\mathcal{U}_{M}$ denote the class of inputs for which $|\mu|_{\left[t_{0}, \infty\right)} \leq M$, that is, the total variation of the inputs is bounded by some constant $M>0$. The following corollary shows that $\mathcal{U}_{M} \subset \mathcal{U}_{c, \theta}$ for some $c>0$, and $\theta \in \mathcal{K}_{\infty}$.

Corollary 6 . Suppose that the hypotheses of Theorem 4 hold with $a<0$ in (5b). Then, system (2) is uniformly asymptotically stable over $\mathcal{U}_{M}$.

Proof. To prove the desired result, we derive the constant $c$ and function $\theta$ in (6). Letting $b:=\max \{\bar{b},|\underline{b}|\}$, we note that

$$
\begin{aligned}
\bar{b} \mu^{+}\left(\left[t_{0}, t\right]\right)-\underline{b} \mu^{-}\left(\left[t_{0}, t\right]\right) & \leq b\left(\mu^{+}\left(\left[t_{0}, t\right]\right)+\mu^{-}\left(\left[t_{0}, t\right]\right)\right) \\
& =b|\mu|_{\left[t_{0}, t\right] .}
\end{aligned}
$$

Since the total variation is non-decreasing with $t$ and is bounded by $M$ for all $t \geq t_{0}$, we can choose $c:=b M$ and $\theta(t):=-a t$ to see that the inequality (6) indeed holds.

Example 2. Consider a bilinear system

$$
d x=A x d t+B x d u
$$

with the matrix $A$ assumed to be Hurwitz. There exists a symmetric positive definite matrix $P>0$ such that $A^{\top} P+P A=-a P$ for some $a>0$. With $V=x^{\top} P x$, this gives $\langle\nabla V(x), A x\rangle \leq-a V(x)$ for all $x \in \mathbb{R}^{n}$. Moreover, we have $\underline{b} V(x) \leq\langle\nabla V(x), B x\rangle \leq \bar{b} V(x)$ for $\underline{b}=\frac{\lambda_{\min }(\tilde{B})}{\lambda_{\max }(P)}, \bar{b}=$ $\frac{\lambda_{\max }(\tilde{B})}{\lambda_{\min }(P)}$, where $\tilde{B}=P B+B^{\top} P$, and $\lambda_{\max }(\cdot), \lambda_{\min }(\cdot)$ denote the maximum and minimum eigenvalues of a matrix, respectively. If $\lambda_{\min }(\tilde{B})>0$ and $\mathcal{U}_{e d}$ comprises of inputs that are eventually non-increasing ${ }^{1}$ then system (10) is asymptotically stable over $\mathcal{U}_{e d}$ because, for each $u(\cdot) \in \mathcal{U}_{e d}$, the term $\mu^{+}\left(\left[t_{0}, t\right]\right)$ becomes constant after some large $t$. Similarly, if $\lambda_{\max }(\tilde{B})<0$ and $\mathcal{U}_{e i}$ comprises of inputs that are eventually non-decreasing then system (10) is asymptotically stable over $\mathcal{U}_{e i}$ because $\mu^{-}\left(\left[t_{0}, t\right]\right)$ becomes constant after some $t$ large enough, for each $u(\cdot) \in \mathcal{U}_{e i}$.

\footnotetext{
1 A function $h: \mathbb{R} \rightarrow \mathbb{R}$ is called eventually non-increasing if there exists $T$ such that for every $\varepsilon_{2}>\varepsilon_{1} \geq 0$, it holds that $h(T+$ $\left.\varepsilon_{2}\right) \leq h\left(T+\varepsilon_{1}\right)$. Similarly, $h(\cdot)$ is called eventually non-decreasing if $-h(\cdot)$ is eventually non-increasing.
} 


\subsection{Input-to-State Stability with Variation}

In contrast to asymptotic stability for a specific class of inputs, we are now interested in studying another notion of stability for system (2). The motivation is to consider systems which are not necessarily asymptotically stable but the maximum value of the state trajectories depends on some norm of the driving input. This may be particularly interesting when $u(\cdot)$ models additive noise or there are undesired impulsive perturbations in the state trajectory.

Definition 7. System (2) is called input-to-state stable (ISS) with respect to variation of the input $u(\cdot)$ if there exist a class $\mathcal{K} \mathcal{L}$ function $\beta$ and a class $\mathcal{K}_{\infty}$ function $\gamma$ such that

$$
|x(t)| \leq \beta\left(\left|x\left(t_{0}\right)\right|, t-t_{0}\right)+\gamma\left(|\mu|_{\left[t_{0}, t\right]}\right) \quad t \geq t_{0} .
$$

Next, we present sufficient conditions under which the property (11) holds.

Theorem 8. Suppose that there exist a continuously differentiable function $V: \mathbb{R}^{n} \rightarrow \mathbb{R}_{+}$, some class $\mathcal{K}_{\infty}$ functions $\alpha_{1}, \alpha_{2}$, and some positive constants $a, b, c>0$ such that for each $x \in \mathbb{R}^{n}$ :

$$
\begin{aligned}
\alpha_{1}(|x|) \leq V(x) & \leq \alpha_{2}(|x|), \\
\langle\nabla V(x), f(x)\rangle & \leq-a V(x), \\
-b V(x)-c \leq\langle\nabla V(x), g(x)\rangle & \leq b V(x)+c,
\end{aligned}
$$

then system (2) is ISS with respect to the variation of $u(\cdot)$.

Proof. Over an interval $\left(t_{i}, t_{i+1}\right)$ between two discontinuities of the input $u(\cdot)$, we have

$$
\begin{aligned}
d V & =\langle\nabla V(x), f(x)\rangle d t+\langle\nabla V(x), g(x)\rangle d u \\
& \leq-a V d t+b V d u+c d u .
\end{aligned}
$$

Using Lemma 10 in Appendix A, the following holds for each $t \in\left(t_{i}, t_{i+1}\right)$ :

$$
V(t) \leq e^{-a\left(t-t_{i}\right)+b|\mu|_{\left[t_{i}, t\right)}} V\left(t_{i}\right)+c e^{b|\mu|_{\left[t_{i}, t\right)}}|\mu|_{\left[t_{i}, t\right)} .
$$

To obtain an upper bound on $V\left(t_{i}^{+}\right)$at a jump instant $t_{i}$, we follow the same arguments as in the proof of Theorem 4 and use the fact that $x\left(t_{i}^{+}\right)=z\left(\mu^{+}\left(\left\{t_{i}\right\}\right)+\mu^{-}\left(\left\{t_{i}\right\}\right)\right)$ where $z(\cdot)$ is such that $\dot{z}(s)=g(z(s))$ for $s \in\left[0, \mu^{+}\left(\left\{t_{i}\right\}\right)\right]$ and $\dot{z}(s)=-g(z(s))$ for $z \in\left[\mu^{+}\left(\left\{t_{i}\right\}\right), \mu^{+}\left(\left\{t_{i}\right\}\right)+\mu^{-}\left(\left\{t_{i}\right\}\right)\right]$, with initial condition $z(0)=x\left(t_{i}^{-}\right)$. Letting $v(s)=$ $V(z(s))$, we have $\dot{v}(s) \leq b v(s)+c$ for $s \in\left[0, \mu^{+}\left(\left\{t_{i}\right\}\right)+\right.$ $\left.\mu^{-}\left(\left\{t_{i}\right\}\right)\right]$ using (12c), and the comparison lemma yields:

$$
v\left(|\mu|_{\left\{t_{i}\right\}}\right) \leq e^{b|\mu|_{\left\{t_{i}\right\}}} v(0)+\frac{c}{b}\left(e^{b|\mu|_{\left\{t_{i}\right\}}}-1\right)
$$

where we recall that $|\mu|_{\left\{t_{i}\right\}}=\mu^{+}\left(\left\{t_{i}\right\}\right)+\mu^{-}\left(\left\{t_{i}\right\}\right)$. This, in turn gives

$$
V\left(t_{i}^{+}\right) \leq e^{b|\mu|_{\left\{t_{i}\right\}}} V\left(t_{i}^{-}\right)+\frac{c}{b}\left(e^{b|\mu|_{\left\{t_{i}\right\}}}-1\right) .
$$

Using these expressions for continuous and discrete flow of the function $V(\cdot)$, it is observed that for any $t \in\left(t_{i}, t_{i+1}\right)$, $i \geq 1$, we have

$$
V(t) \leq V_{i c}(t)+V_{u_{c}}(t)+V_{u_{d}}(t)
$$

where $V_{i c}(\cdot)$ denotes the evolution of $V(\cdot)$ due to the initial condition $V\left(t_{0}\right), V_{u_{c}}(\cdot)$ denotes the evolution due to continuous part of the input between discontinuities, and $V_{u_{d}}$ denotes the evolution due to jumps in the input. The values of these three functions are given by:

$$
V_{i c}(t)=e^{-a\left(t-t_{0}\right)+b|\mu|_{\left[t_{0}, t\right)}} V\left(t_{0}\right)
$$

$$
\begin{aligned}
V_{u_{c}}(t)= & c \sum_{k=0}^{i-1} e^{-a\left(t-t_{k+1}\right)+b|\mu|_{\left(t_{k}, t\right)}}|\mu|_{\left(t_{k}, t_{k+1}\right)} \\
& +c e^{b|\mu|_{\left(t_{i}, t\right)}|\mu|_{\left(t_{i}, t\right)}} \\
V_{u_{d}}(t)= & \frac{c}{b} \sum_{k=1}^{i} e^{-a\left(t-t_{k}\right)+b|\mu|_{\left(t_{k}, t\right)}}\left(e^{b|\mu|_{\left\{t_{k}\right\}}}-1\right) .
\end{aligned}
$$

Using $e^{-a\left(t-t_{k}\right)} \leq 1$ and $e^{b|\mu|_{\left(t_{k}, t\right)}} \leq e^{b|\mu|_{\left[t_{0}, t\right]}}$ for each $k \leq i$, we obtain

$$
V_{u_{d}} \leq \frac{c}{b} e^{b|\mu|_{\left[t_{0}, t\right]}} \sum_{k=1}^{i}\left(e^{b|\mu|_{\left\{t_{k}\right\}}}-1\right) .
$$

To obtain similar bound for $V_{u_{c}}(t)$, we multiply and divide (14) by $b$, and in addition use the fact $r \leq e^{r}-1$, for $r \geq 0$, to get

$$
\begin{aligned}
V_{u_{c}}(t) \leq \frac{c}{b} e^{b|\mu|_{\left[t_{0}, t\right]}} & \left(\sum_{k=0}^{i-1}\left(e^{b|\mu|_{\left(t_{k}, t_{k+1}\right)}}-1\right)\right. \\
& \left.+\left(e^{b|\mu|_{\left(t_{i}, t\right)}}-1\right)\right)
\end{aligned}
$$

Adding (15) and (16), and using the fact $\left(e^{r_{1}}-1\right)+\left(e^{r_{2}}-\right.$ $1) \leq e^{r_{1}+r_{2}}-1$, we have

$$
V_{u_{c}}(t)+V_{u_{d}}(t) \leq \frac{c}{b} e^{b|\mu|_{\left[t_{0}, t\right]}}\left(e^{b|\mu|_{\left[t_{0}, t\right]}}-1\right) .
$$

Finally, the upper bound on $V_{i c}(t)$ is obtained as follows:

$$
\begin{gathered}
V_{i c}(t)=e^{-a\left(t-t_{0}\right)} V\left(t_{0}\right)+e^{-a\left(t-t_{0}\right)}\left(e^{b|\mu|_{\left[t_{0}, t\right]}}-1\right) V\left(t_{0}\right) \\
\leq e^{-a\left(t-t_{0}\right)} V\left(t_{0}\right)+\frac{1}{2}\left(e^{-a\left(t-t_{0}\right)} V\left(t_{0}\right)\right)^{2} \\
\quad+\frac{1}{2}\left(e^{b|\mu|_{\left[t_{0}, t\right]}}-1\right)^{2} .
\end{gathered}
$$

The estimate as in (11) can now be computed by introducing the following functions:

$$
\beta(r, t):=\alpha_{1}^{-1}\left(\hat{\theta}\left(e^{-a t} \alpha_{2}(r)\right)\right)
$$

where $\hat{\theta}(r):=r+\frac{1}{2} r^{2}$ is a class $\mathcal{K}_{\infty}$ function, and

$$
\gamma(s):=\frac{1}{2}\left(e^{b s}-1\right)^{2}+\frac{c}{b} e^{b s}\left(e^{b s}-1\right) .
$$

Plugging (17), (18) in (13) and noting that $V\left(x\left(t_{0}\right)\right) \leq$ $\alpha_{2}\left(\left|x\left(t_{0}\right)\right|\right)$ and $|x(t)| \leq \alpha_{1}^{-1}(V(x(t)))$, the desired result (11) is obtained with $\bar{\beta}$ and $\gamma$ defined in (19) and (20), respectively.

Remark 9. For absolutely continuous functions, the variation is equal to $\mathcal{L}^{1}$-norm of the derivative of that function. Since system (2), in that case, could be thought of as driven by the derivative of the input, the notion of ISS given in Definition 7 coincides with the notion of integral ISS dicussed in Sontag [1998]. Comparing our sufficient conditions with the ones presented in that paper, we chose to work with a linear multiple of the norm of the input instead of an arbitrary class $\mathcal{K}_{\infty}$ function so that the resulting inequalities involving measures could be explicitly solved.

Example 3. Consider the MDE $d x=-x+d u$ with the Lyapunov function $V(x)=\frac{1}{2} x^{2}$. It is easy to see that the inequalities (12) hold with $\alpha_{1}(r)=\alpha_{2}(r)=\frac{1}{2} r^{2}$, and the constants $a=b=2, c=1$. Thus the system is ISS with respect to variation.

Example 4. Consider the following bilinear system:

$$
d x=A x d t+\left(B_{1} x+B_{2}\right) d u
$$


with $A$ being a Hurwitz matrix. We show that this system is ISS with respect to variation of the input. To see this, we consider the Lyapunov function $V=x^{\top} P x$ where $P$ is a symmetric positive definite matrix satisfying $A^{\top} P+$ $P A=-a P$ for some $a>0$. Thus, (12a) and (12b) hold. To find the desired constants in $(12 \mathrm{c})$, note that

$$
\left\langle 2 P x, B_{1} x\right\rangle \leq 2\left\|P B_{1}\right\| \cdot|x|^{2}
$$

where $\left\|P B_{1}\right\|$ denotes the induced Euclidean norm of the matrix $P B_{1}$. Also,

$$
\left\langle 2 P x, B_{2}\right\rangle \leq 2\left\|P B_{2}\right\| \cdot|x| \leq\left\|P B_{2}\right\|^{2}+|x|^{2}
$$

Similarly, by using the Cauchy-Schwarz inequality in other direction, we get

$$
\left\langle 2 P x, B_{1} x+B_{2}\right\rangle \geq-\left(2\left\|P B_{1}\right\|+1\right)|x|^{2}-\left\|P B_{2}\right\|^{2} .
$$

Using the fact $|x|^{2} \leq \frac{1}{\lambda_{\min (P)}} V(x)$, it is observed that (12c) holds with

$$
b=\frac{2\left\|P B_{1}\right\|+1}{\lambda_{\min }(P)}, \quad c=\left\|P B_{2}\right\|^{2} .
$$

\section{CONCLUSION}

In this paper, we studied the problem of stability in a class of differential equations driven linearly by differential measures associated with functions of locally bounded variation. Sufficient conditions based on Lyapunov function were given for uniform asymptotic stability over a certain class of inputs. We then studied a generalized notion of integral ISS in measure driven systems and presented sufficient conditions under which the supremum norm of the state is bounded by the total variation of the measure associated with input.

\section{Appendix A. GENERALIZED COMPARISON LEMMA}

Lemma 10. Consider a continuous function $V(\cdot)$ that satisfies

$$
d V \leq-a V d t+b V d \mu+c d \mu
$$

on an interval $\left[t_{0}, t_{1}\right)$, then for each $t \in\left[t_{0}, t_{1}\right)$

$$
V(t) \leq e^{-a\left(t-t_{0}\right)+b \mu\left(\left[t_{0}, t\right)\right)} V\left(t_{0}\right)+c e^{b|\mu|_{\left[t_{0}, t\right)}}|\mu|_{\left[t_{0}, t\right)} .
$$

Proof. We split the proof in two steps:

Step 1. Consider a continuous function $W$ such that

$$
d W=-a W d t+b W d \mu+c d \mu
$$

with $W\left(t_{0}\right)=V\left(t_{0}\right)$. Let us consider a sequence $\left\{u_{k}\right\}_{k=1}^{\infty}$ of continuously differentiable functions converging to $u(\cdot)$ with respect to $\mathcal{L}^{1}$-norm and let $W_{k}$ represent the solution of (A.2) obtained by replacing $d \mu$ with $\dot{u}_{k} d t$. Then

$$
W_{k}(t)=\Phi\left(t, t_{0}\right) W_{k}\left(t_{0}\right)+c \int_{t_{0}}^{t} \Phi(t, s) \dot{u}_{k}(s) d s
$$

where $\Phi(t, s)=e^{-a(t-s)+b\left(u_{k}(t)-u_{k}(s)\right)}$ and $W_{k}\left(t_{0}\right)=$ $W\left(t_{0}\right)$ for each $k \geq 1$. Using the inequalities $e^{-a(t-s)} \leq 1$ and $\left|u_{k}(t)-u_{k}(s)\right| \leq \int_{t_{0}}^{t}\left|\dot{u}_{k}(\tau)\right| d \tau$ for each $s \in\left[t_{0}, t\right]$, we obtain

$$
\begin{aligned}
& W_{k}(t) \leq e^{-a\left(t-t_{0}\right)+b\left(u_{k}(t)-u_{k}\left(t_{0}\right)\right)} W_{k}\left(t_{0}\right) \\
& +c e^{b\left\|\dot{u}_{k\left[t_{0}, t\right)}\right\|_{1}}\left\|\dot{u}_{k\left[t_{0}, t\right)}\right\|_{1} .
\end{aligned}
$$

From [Ambrosio et al., 2000, Theorem 3.9], one can pick the sequence $u_{k}(\cdot)$ converging to $u(\cdot)$ in $\mathcal{L}^{1}$-norm such that $\left\|\dot{u}_{k\left[t_{0}, t\right)}\right\|_{1}$ converges to $|\mu|_{\left[t_{0}, t\right)}$. Hence,

$$
W(t) \leq e^{-a\left(t-t_{0}\right)+b \mu\left(\left[t_{0}, t\right)\right)} W\left(t_{0}\right)+c e^{|\mu|_{\left[t_{0}, t\right)}}|\mu|_{\left[t_{0}, t\right)} .
$$

Step 2. We now show that $V(t) \leq W(t)$ for each $t \in\left[t_{0}, t_{1}\right)$, from which the desired result follows. Indeed, if $V$ and $W$ satisfy (A.1) and (A.2), then $\bar{W}:=V-W$ satisfies

$$
d \bar{W} \leq-a \bar{W} d t+b \bar{W} d \mu
$$

where $\bar{W}\left(t_{0}\right)=0$. Using the same arguments as in the proof of Theorem 4 , we obtain

$$
\bar{W}(t) \leq e^{-a\left(t-t_{0}\right)+b \mu\left(\left[t_{0}, t\right]\right)} \bar{W}\left(t_{0}\right)
$$

so that $\bar{W}(t) \leq 0$, or equivalently $V(t) \leq W(t)$.

\section{REFERENCES}

V. Acary, B. Brogliato, and D. Goeleven. Higher order Moreau's sweeping process: Mathematical formulation and numerical simulation. Mathematical Programming Ser. A, 113:133 - 217, 2008.

L. Ambrosio, N. Fusco, and D. Pallara. Functions of Bounded Variation and Free Discontinuity Problems. Clarendon Press, Oxford, 2000.

A. Bressan. On differential systems with impulsive controls. Rendiconti del Seminario Matematico della Università di Padova, 78:227 - 235, 1987.

A. Bressan and F. Rampazzo. On differential systems with quadratic impulses and their applications to Lagrangian mechanics. SIAM J. Control \& Optim., 31(5):1205 1220, 1993.

A. Bressan Jr. and F. Rampazzo. Impulsive control systems with commutative vector fields. J. Optimization Theory and Applications, 71, 1991.

B. Brogliato. Nonsmooth Mechanics. Springer-Verlag, London, second edition, 1999.

W. J. Code and G. N. Silva. Closed loop stability of measure-driven impulsive control systems. J. Dynamical and Control Systems, 16(1):1 - 21, 2010.

J. P. Hespanha, D. Liberzon, and A. Teel. Lyapunov conditions for input-to-state stability of impulsive systems. Automatica, 44:2735 - 2744, 2008.

H. K. Khalil. Nonlinear Systems. Prentice-Hall, Inc., 3rd edition, 2002.

J. Kurzweil. Generalized ordinary differential equations. Czechoslovak Mathematical Journal, 8(3):360 - 388, 1958.

M. D. P. Monteiro Marques. Differential Inclusions in Nonsmooth Mechanical Problems: Shocks and Dry Friction, volume 9 of Progress in Nonlinear Differential Equations and their Applications. Birkhäuser, 1993.

J. J. Moreau and M. Valadier. A chain rule involving vector functions of bounded variation. Journal of Functional Analysis, 74(2):333 - 345, 1987.

W. W. Schmaedeke. Optimal control theory for nonlinear vector differential equations containing measures. SIAM J. Control, 3(2):231 - 280, 1965.

E. D. Sontag. Smooth stabilization implies coprime factorization. IEEE Trans. on Automatic Control, AC-34 (4):435 - 443, 1989.

E. D. Sontag. Comments on integral variants of ISS. Systems and Control Letters, 34:93-100, 1998.

H. J. Sussmann. On the gap between deterministic and stochastic ordinary differential equations. The Annals of Probability, 6(1):19-41, 1978. 